\title{
Strict Anatomical Colocalization of Vitiligo and Elastolytic Granulomas
}

\author{
N. Merino de Paz ${ }^{\mathrm{a}} \quad$ M. Rodríguez-Martín ${ }^{\mathrm{a}}$ \\ M. García Bustínduy ${ }^{a} \quad$ A. Martín-Herrera ${ }^{b}$
}

A. Noda-Cabrera ${ }^{a}$

Departments of a Dermatology and ${ }^{b}$ Pathology, Hospital Universitario de Canarias, University of La Laguna, La Laguna, Spain

\section{Key Words}

Vitiligo Elastolytic granuloma $\cdot$ Phototherapy

\section{Abstract}

Vitiligo is the most common depigmenting disorder, with a worldwide occurrence of $0.1-2 \%$ in the general population. Multiple conditions have been described colocalized in vitiligo patches, like psoriasis or lichen planus. However, actinic granuloma has not been described in association with vitiligo lesions so far.

\section{Introduction}

Vitiligo is the most common depigmenting disorder, with a worldwide occurrence of $0.1-2 \%$ in general population [1]. Multiple conditions have been described colocalized in vitiligo patches, like psoriasis or lichen planus [2-5]. However, actinic granuloma has not been described in association with vitiligo lesions so far.

Actinic granuloma was first described by O’Brien in 1975 as an uncommon disease, most frequently presented in middle-aged women $[6,7]$. Its classification and pathogenesis is controversial. Some authors consider actinic granuloma a subtype of annular granuloma and others a different entity [6-9]. The pathogenesis of this condition is still uncertain [7-9].

\section{Case Report}

We report the case of a 50-year-old woman, who was referred to our Dermatology Department in 2006 with a diagnosis of lip-tip vitiligo. The milky patches were located in the face, on the dorsum of the hands, and on the forearms and legs. Her past medical history was only remarkable for hyperthyroidism 
diagnosed 1 year ago. There was no other family history of dermatologic or endocrine problems. No diagnosis of lupus, sarcoidosis or other dermatologic diseases could be elucidated in her medical or family history. She was treated with topical steroids (Clovate ${ }^{\circledR}$ cream) and topical tacrolimus (Protopic ${ }^{\circledR}$ $0.1 \%$ ointment), with poor response in achral lesions. Oral PUVA therapy with 8-MOP was started in 2007, obtaining a moderate repigmentation in distal areas. Treatment was given 3 times weekly for a maximum of 20 weeks. In June 2009, during a routine follow-up, the patient complained that non-pruriginous lesions had appeared in her hands 2 weeks ago. On physical examination, she presented with multiple asymptomatic, erythematous, annular plaques and solitary papules $5-10 \mathrm{~mm}$ in diameter, located on the dorsum of both hands, strictly confined to vitiliginous lesions. No other lesions in different areas could be observed. Full blood count, liver and renal function and urine analysis were all in normal ranges. Based on the clinical picture, presumptive diagnosis of coincident vitiligo and annular granuloma was made. A skin biopsy was performed.

Histological examination revealed mono- and multinucleated histiocytes and inflammatory infiltrates in the dermis. Deformed and fragmented elastic fibers in the dermis were also revealed using Orcein staining. Fibers phagocyted by the histiocytes were seen, too. Besides, absence of melanocytes in Fontana-Masson staining was demonstrated. These histological findings confirmed the coexistence of vitiligo and elastolytic or actinic cutaneous granuloma.

\section{Conclusion}

Several articles have suggested that annular and actinic granuloma are different conditions based on histopathological characteristics [8]. Different terms have been used to designate this disorder: annular elastolytic giant-cell granuloma (AEGCG) [9], O’Brien granuloma [7], multiple $[10,11]$, actinic [7, 8] or elastolytic [12] granuloma. Some articles suggest that actinic granuloma is a subtype of AEGCG [12]. The term 'multiple granuloma' was coined by Leiker in 1964, and it is clinically similar to actinic granuloma, being considered the same entity in some studies. Nevertheless, multiple granuloma presents a distinctive histological finding of focal necrobiosis. A photo-mediated etiology has been proposed in the literature for this subtype $[10,11]$.

The etiology of actinic granuloma remains obscure. However, several theories have been postulated. O'Brien suggested the role of infrared and ultraviolet actinic radiation [6, $7,13]$. The importance of a granulomatous chronic inflammation in association with another systemic illness such as sarcoidosis or diabetes mellitus was proposed by Hanke [9].

The actinic damage hypothesis is based on the cutaneous cell-mediated immune response. CD4 lymphocytes would act against the solar-damaged elastic fibers, which could be recognized as antigenic products $[6,7,13]$. Histological characteristics like sarcoidal granuloma or multinucleated giant cells suggest an inflammatory response to small products, like altered elastic fibers. The confinement of the inflammatory reaction to the superficial dermis, corresponding to the area of solar elastosis, supports the actinic theory [8].

This photo-mediated damage could be considered as a trigger factor in our patient and could explain the strict colocalization of elastolytic granuloma on vitiligo patches. Vitiliginous skin is more photo-sensible and the actinic damage is more intense than in the rest of the body [14]. In this case, the affected skin on the dorsum of the hands was not only prone to photo-damage as a result of sun exposure, but it was also selectively exposed to PUVA therapy over the last 2 years in our patient.

In this case, we present an uncommon clinical feature with strict colocalization of elastolytic granuloma in vitiliginous areas. The location of the lesions strongly supports 
the photo-damage hypothesis in our patient. Moreover, the patient received PUVA and sun therapy as an important part of her treatment.

To our knowledge, this is the first patient with coexistence and strict anatomical coincidence of actinic granuloma and vitiligo reported in the literature thus far. We conclude that the actinic radiation played an important role in this form of granuloma in our patient. However, further studies to clarify pathogenetic links between vitiligo and actinic granuloma would be necessary.

Fig. 1. Vitiliginous areas with coincident multiple small papules located on the dorsum and lateral aspect of both hands. The annular plaques only affect depigmented areas.

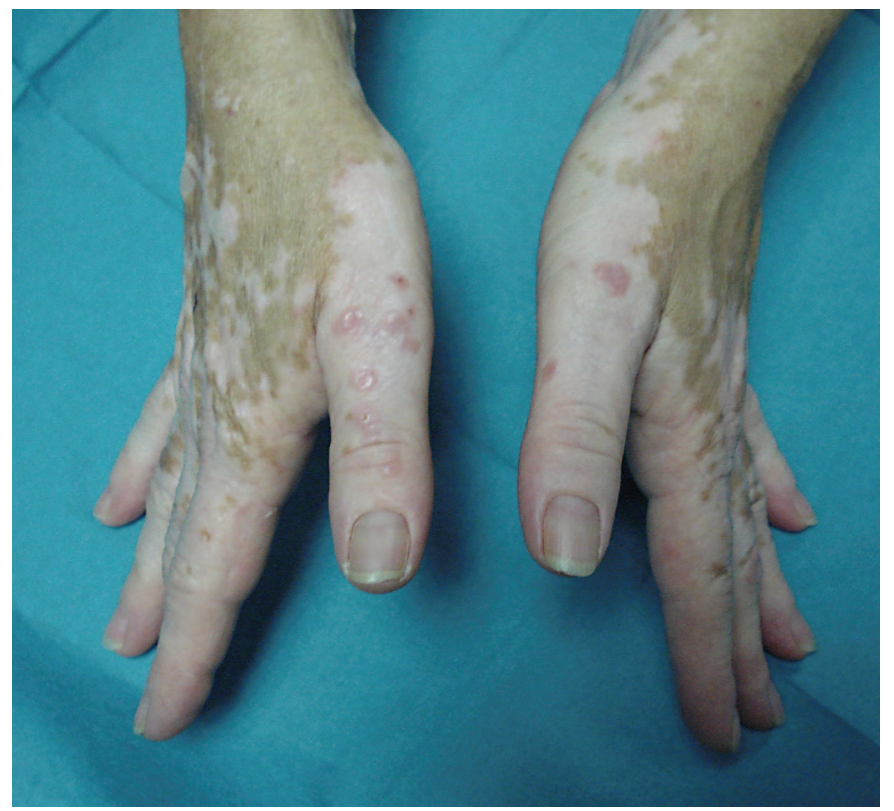


Fig. 2. Histological examination revealed mono- and multinucleated histiocytes and inflammatory infiltrates in the dermis. Deformed and fragmented elastic fibers in the dermis were also revealed (Orcein staining, 100 $\times$ ).

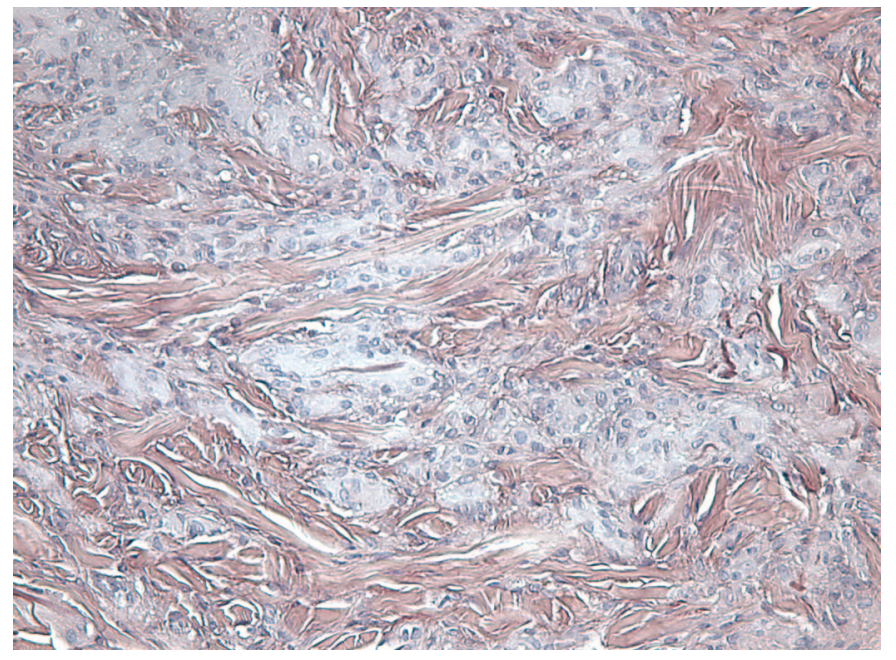




\section{References}

1 Halder RM, Chappell JL: Vitiligo update. Semin Cutan Med Surg 2009;28:86-92.

-2 Berger TG, Kiesewetter F, Maczek C, Bauer N, Lueftl M, Schuler G, Simon M Jr: Psoriasis confined strictly to vitiligo areas - a Koebner-like phenomenon? Eur Acad Dermatol Venereol 2006;20:178-183.

-3 Rodríguez-Martín M, Sáez-Rodríguez M, Carnerero-Rodríguez A, Cabrera de Paz R, Sidro-Sarto M, Pérez-Robayna N, Sánchez R, García-Bustínduy M, MartínHerrera A, Noda-Cabrera A: Coincidental presentation of vitiligo and psoriasis in a patient with polyglandular autoimmune syndrome. Clin Exp Dermatol 2007;32:453.

-4 Inamadar AC, Sampagavi VV, Athanikar SB, Patil MN, Deshmukh NS: Vitiligo and psoriasis: coexistence with colocalization. Indian J Dermatol Venereol Leprol 2001;67:214-215.

5 Gül U, Soylu S, Demiriz M: Colocalization of lichen planus and vitiligo associated with selective IgA deficiency. Skinmed 2007;6:202-203.

6 Stein JA, Fangman B, Strober B: Actinic granuloma. Dermatol Online J 2007 Jan 27;13:19.

7 O'Brien JP: Actinic granuloma: an annular connective tissue disorder affecting sun- and heat-damaged (elastotic) skin. Arch Dermatol 1975;111:460-466.

8 Al-Hoqail I, Al-Ghamdi A, Martinka M, Crawford RI: Actinic granuloma is a unique and distinct entity. Am J Dermatoph 2002;24:209-212.

-9 Hanke CW, Bailin PL, Roenigk HH Jr: Annular elastolytic giant cell granuloma. A clinicopathologic study of five cases and a review of similar entities. J Am Acad Dermatol 1979;1:413-421.

10 Sandhu K, Saraswat A, Gupta S, Shukla R, Handa S: Granuloma multiforme. Int J Dermatol 2004;43 441-443.

11 Kumari R, Thappa DM, Chougule A, Adityan B: Granuloma multiforme: a report from India. Indian J Dermatol Venereol Leprol 2009;75:296-299.

12 Limas C: The spectrum of primary cutaneous elastolytic granulomas and their distinction from granuloma annulare: a clinicopathological analysis. Histopathology 2004;44:277-282.

13 Regan W: Letter. Int J Dermatol 2006;45:1001.

14 Amblard P, Leccia MT: Skin diseases with photosensitivity. Rev Prat 1992;42:1365-1368. 